\title{
CORRESPONDENCE
}

\section{The Role of the Complement System in Age-Related Macular Degeneration}

by Prof. Dr. rer. nat. Bernhard H. F. Weber,

Prof. Dr. med. Peter Charbel Issa, Dr. rer. nat. Diana Pauly,

Dr. med. Philipp Herrmann, Felix Grassmann, MSc,

Prof. Dr. med. Frank G. Holz in volume 8/2014

\section{Mitochondrial DNA Plays an Important Role}

In their paper on the pathogenesis of age-related macular degeneration (AMD), the authors (1) do not mention that mitochondrial DNA (mtDNA) plays an important role in the etiology of AMD. AMD is more than a possibly complement-associated disease accompanied by nuclear DNA (nDNA) dysfunction. While the article makes reference to the genetics of gene defects in the nucleus, the mitochondrial abnormalities associated with AMD are not discussed.

In the meantime, there is growing evidence that defects caused by oxidative stress along the respiratory chain in the mitochondria play a greater role by far in the pathogenesis of AMD than previously thought. Especially oxidative stress due to an apparently genetically determined reduced ability to inactivate highly reactive oxygen species (ROS) in the mitochondria seems to lead to significant mtDNA damage, above all in AMD patients (2). In addition, the DNA repair mechanisms in AMD patients appear to be significantly more affected on a mitochondrial level than on a nuclear DNA (nDNA) level (3). These insights may lead to new treatment options with the potential to prevent the deleterious symptoms in the late stages of AMD by improving metabolic processes on a mitochondrial level early in the disease, even prior to the possible activation of complement-associated pathomechanisms in full-blown late-stage AMD.

Taking mitochondrial processes into account is crucial to developing a deeper understanding of AMD in humans. Awareness of the role of mitochondrial dysfunction in the early stages of $\mathrm{AMD}$ is a prerequisite for establishing adequate treatments - before the manifestation of the deleterious symptoms of AMD in the patient.

DOI: 10.3238/arztebl.2014.0366a

\section{REFERENCES}

1. Weber BHF, Charbel Issa P, Pauly D, Herrmann P, Grassmann F, Holz FG:The role of the complement system in age-related macular degeneration. Dtsch Arztebl Int 2014; 111: 133-8

2. Blasiak J, Glowacki S, Kauppinen A, Kaarniranta K: Mitochondrial and nuclear DNA damage and repair in age-related macular degeneration. Int J Mol Sci 2013; 14: 2996-3010.

3. Jarrett SG, Lin H, Godley BF, Boulton ME: Mitochondrial DNA damage and its potential role in retinal degeneration. Prog Retin Eye Res 2008; 27: 596-607.

\section{In Reply}

We would like to thank Mr. Uebermuth for highlighting the importance of mitochondrial dysfunction in the pathogenesis of AMD. To understand this multifactorial disease it is, of course, essential to take its complex etiology into consideration which is characterized by so far poorly understood interactions between multiple genetic and non-genetic factors contributing to the transition from a healthy to a diseased retina. Such factors include, among others, polymorphisms of the mitochondrial DNA occurring as defined haplogroups in the population; their modifying effects on nuclear genetic factors are being discussed at present (1). Interestingly, these new studies are finding that such factors also have effects on the expression of the nuclear genes of the alternative complement cascade, leading to a paradigm change in our understanding of mitochondrial-nuclear interactions and, at the same time, are highlighting the key role of the innate immune system in AMD.

Due to the topic's complexity, we had to focus our article (2) to one aspect of AMD research, which, however, has already opened the way to first therapeutic interventions being evaluated in clinical studies. However, we are aware that recent scientific findings point towards further promising aspects of AMD pathogenesis (such as oxidative damage, lipofuscin accumulation, chronic inflammation), adding new important target structures for the development of future therapies.

DOl: 10.3238/arztebl.2014.0366b

\section{REFERENCES}

1. Cristina Kenney M, Chwa M, Atilano SR, et al.: Inherited mitochondrial DNA variants can affect complement, inflammation and apoptosis pathways: insights into mitochondrial-nuclear interactions. Hum Mol Genet 2014; 4: [Epub ahead of print]

2. Weber BHF, Charbel Issa P, Pauly D, Herrmann P, Grassmann F, Holz FG:The role of the complement system in age-related macular degeneration. Dtsch Arztebl Int 2014; 111: 133-8.

On behalf of the authors:

Prof. Dr. rer. nat. H. F. Bernhard

Weber Institute of Human Genetics

University of Regensburg, Germany

Prof. Dr. med. Frank G. Holz

Department of Ophthalmology

University of Bonn, Germany

Frank.Holz@ukb.uni-bonn.de

\section{Conflict of interest statement}

Prof. Weber has received fees for consultancy and presentations, as well as reimbursements for travel costs by Alcon and Novartis.

Prof. Holz has received fees for consultancy and presentations by Acucela, Alcon, Allergan, Genentech, Novartis, Roche, Merz, Heidelberg Engineering, and Bayer. He has received reimbursements for travel costs from Roche, Novartis, Genentech, Acuela, and Pfizer. 